\title{
THERMAL INDUCED MICROSTRUCTURES OF KOH ETCHED SILICON SURFACE
}

\author{
Shobha Kanta Lamichhane \\ Tribhuvan University, Prithwi Narayan Campus, Pokhara \\ Corresponding address: sklamichhane@ hotmail.com \\ Received 6 November, 2008; Revised 28 December, 2008
}

\begin{abstract}
Anisotropic $\mathrm{KOH}$ etching of silicon for the fabrication of Micro-electro mechanical system (MEMS) part is based on surface finish and angular dependence of etch rate, creating thin diaphragm. The absolute values of orientation dependent etch rate is found to vary with thermal agitation. In this work, experimental results of etch rate is found quite consistent with simulated and are justify with their unusual values of activation energy along different planes. The various sites that an atom can occupy are not equivalent of their energy; some are more favorable to removal than others. In this paper attention is given to demonstrate thermal activation energy is the prime parameter that influences the behavior of etching mechanism as well as AFM surface morphology. Lowvoltage contact mode atomic force microscopy (AFM) has been employed to analyze the morphology of the etched silicon surface at relevant different temperature. A systematic variation in morphological growth leads to stabilized surface structure under the influence of associated activation energy is concluded.
\end{abstract}

Key words: Etching, Anisotropy, Etch rate, diaphragm, MEMS, LPCVD, SOI, AFM

\section{INTRODUCTION}

MEMS devices are typically made from silicon (Kovacs, 1998) using conventional semiconductor manufacturing techniques. They can be either etched from larger structure or built up by employing material deposition processes (Finne and Klein, 1967). Silicon micromachining technology has become popular for the fabrication of a number of MEMS devices. Silicon micromachining provides proper dimensional control and consistent mechanical properties and is now established as a mature technology for micro devices. It allows us to control precisely the shape and dimension of microstructure (Finne and Klein 1967; Campbell et al. 1995; Abott, 1993; Schwartz and Robbin, 1961). The technology is now being used to fabricate sensors for measuring pressure, force, flow and acceleration using the advantage of batch processing.

Etching silicon strongly depends on orientation of crystal plane and hence anisotropy. Various experimental techniques have been developed to study anisotropic etching of silicon. Etching silicon can perform with various etchants, such as KOH, TMAH and EDP are few examples (Abott, 1993; Campbell et al., 1995; Schwartz and Robbin, 1961; Kenneth, 1978). The dissolution of silicon is needed for deep etching and micromachining, shaping and cleaning. Taking out silicon atoms from its surface with time for a given temperature and concentration of $\mathrm{KOH}$ is not same for different plane orientations. Its flexibility is exploited to design microstructures and devices with fraction of micron resolution (Schwartz and Robbin, 1961; Lee, 1969) nowadays. 
For a given temperature and concentration, removal of silicon atoms is not same for all silicon crystal planes. The moving surface with time depends on etchant's temperature (Abott, 1993; Campbell et al., 1995; Schwartz and Robbin, 1961; Kenneth 1978) and etchant's concentration (Campbell et al., 1995; Abott, 1993) as well as doping of silicon substrate (Lee, 1969). A high etching rate is generally desirable in a manufacturing environment. Too high etch rate however may render a process difficult to control because during etching, hydrogen bubbles (Finne and Klein, 1967) are formed on silicon surface. Thus, pyramid formations (Finne and Klein 1967; Abott, 1993; Schwartz and Robbin, 1961) can be related to poor bubble detachment, precluding diffusion to specific point on the surface. However, anisotropic etching has provided as a tool for shaping of silicon structures and as an evaluation of protective surface films on silicon substrate (Schwartz and Robbin, 1961; Kenneth, 1978; Lee, 1969). Due to diverse functional dependence of etch rate (ER), a complete study of this system is extremely complicated. So, accuracy of ER may vary from laboratory to laboratory and accuracy level of measurement techniques. In this study, ER of silicon and its morphological microstructure as observed by AFM has been discussed. In particular, etch rate along (111) crystallographic orientation at relevant different temperatures and its variation in surface roughness as observed by AFM is highlighted.

\section{EXPERIMENTAL PROCEDURE}

The silicon wafer of thermally bonded silicon on insulator (SOI) with (100) orientation was used in this study. The thickness of the top silicon buried $\mathrm{SiO}_{2}$ layer and bottom silicon were 2 and $450 \mathrm{~mm}$ respectively. The wafers were of p-type silicon with a resistively of $4-631022 \mathrm{~V}-\mathrm{cm}$ for the top silicon and $12-15 \mathrm{~V}-\mathrm{cm}$ for the bottom silicon. Only the top silicon layer was etched by the KOH solution. First, the SOI wafers were prepared with standard RCA cleaning which consist of two consecutive cleaning solutions (standard clean1, i.e. SC1 clean). Radio Corporation of America, Standard Clean 1 (RCASC1) Cleaning including $\mathrm{H}_{2} \mathrm{O}-\mathrm{H}_{2} \mathrm{O}_{2}-\mathrm{NH}_{4} \mathrm{OH}$ with volume ratio typically 5:1:1 was adopted in many steps during experimentation since it removes organic contaminants and particles physically attached to a wafer surface. A $450 \AA$ layer of $\mathrm{Si}_{3} \mathrm{~N}_{4}$, which acts as a $\mathrm{KOH}$ etching mask, was deposited on each wafer using low pressure chemical vapor deposition (LPCVD). Oxide can be used as an etch mask for short period in the $\mathrm{KOH}$ solution, but for long periods, nitride is a better etch mask as it etches more slowly. The SOI wafer was then cut into $10 \mathrm{~mm} \times 10 \mathrm{~mm}$ pieces. With the dice pieces held on a vacuum chuck, positive photoresist, OCG 825, was used to pattern the $\mathrm{Si}_{3} \mathrm{~N}_{4}$ for the $\mathrm{KOH}$ etching mask. The nitride was etched with CF4 plasma and then the exposed portion of the top silicon on each SOI wafer was etched in $\mathrm{KOH}$ solution of varying temperature and concentration. To assure the sample free of particulate and other airborne contaminants, the experiment were conducted in a dust free room.

Samples prepared in this way, were ready for measurements and microscopic observations by AFM. Images were taken in low voltage contact mode. Before performing AFM measurement, it was ascertained with the help of spirit level and AFM software, scanner plane and AFM head plane were made parallel. During each measurement $0.1 \mu \mathrm{m}$ was taken as scan size. Finally, images were recorded in contact mode AFM. Etch rate, surface roughness (curve for height vs. topographical distance), activation energy of etched surfaces is chosen as the parameter for analysis. 


\section{RESULTS AND DISCUSSIONS}

In this study, 2D moving surface is taken at an ambient condition for understanding etching of silicon. Choosing 'd' spacing as the parameter for their characterization. This can be achieved by consideration on

$$
\frac{1}{d_{h k l}^{2}}=\frac{h^{2}+k^{2}+l^{2}}{a^{2}}
$$

Where 'd' is inter-planer distance, 'a' is lattice parameter and (hkl) are Miller indices. As a result, inter-planer distances are for (100): $5.43{ }^{0} \mathrm{~A}$ and for (110): $3.84{ }^{0} \mathrm{~A}$. Silicon has diamond lattice structure consist of two interpenetrating fcc lattices displaced along the body diagonal of the cube cell by one quarter the length of its diagonal. In (111) silicon, atoms are tetrahedrally bonded. So, interplanear distance would be distance between the apex of the tetrahedron to the base, say $\mathrm{O}$ and $[(\mathrm{a} / 4) \cdot(\vec{x}+\vec{y}+\vec{z})]$, so that distance between two consecutive planes along $(111)$ is $[(\mathrm{a} / 4) \sqrt{3}]=2.35{ }^{0} \mathrm{~A}$. Number of plane removed per second is determined by

$$
\text { Nos of planes etched out per second }=\frac{\text { Etch.rate. } \text { per } \text {. } \min }{60 \times \text { Interplanar.dis } \tan c e}
$$

Time taken for single plane removal is just its reciprocal. Hand on data for ER and data achieved by this technique are given in table 1 below.

\begin{tabular}{|c|c|c|c|c|c|c|c|c|c|c|c|}
\hline \multicolumn{4}{|c|}{ Plane (100) } & \multicolumn{4}{|c|}{ plane (110) } & \multicolumn{4}{|c|}{ plane (111) } \\
\hline $\begin{array}{l}\text { Temp } \\
\left({ }^{0} \mathrm{C}\right)\end{array}$ & $\begin{array}{l}\text { ER } \\
(\mu \mathrm{m} / \mathrm{min})\end{array}$ & A & B & $\begin{array}{l}\text { Temp } \\
\left({ }^{0} \mathrm{C}\right)\end{array}$ & $\begin{array}{l}\text { ER } \\
(\mu \mathrm{m} / \min )\end{array}$ & A & $\mathrm{B}$ & $\begin{array}{l}\text { Temp } \\
\left({ }^{0} \mathrm{C}\right)\end{array}$ & $\begin{array}{c}\text { ER } \\
(\mu \mathrm{m} / \mathrm{min})\end{array}$ & A & B \\
\hline 20 & 0.024 & 00.736 & 1.357 & 20 & 0.035 & 1.519 & 0.6583 & 70 & 0.0052 & 0.368 & 2.7137 \\
\hline 40 & 0.108 & 03.314 & 0.301 & 40 & 0.160 & 6.944 & 0.1440 & 75 & 0.0342 & 2.423 & 0.4125 \\
\hline 60 & 0.410 & 12.582 & 0.079 & 60 & 0.620 & 26.908 & 0.0371 & 80 & 0.0722 & 5.117 & 0.1954 \\
\hline 80 & 1.300 & 39.895 & 0.025 & 80 & 2.000 & 86.801 & 0.0115 & 85 & 0.1672 & 5.131 & 0.1948 \\
\hline 100 & 3.800 & 116.61 & 0.008 & 100 & 5.800 & 251.72 & 0.0039 & 90 & 0.3982 & 2.22 & 0.0818 \\
\hline
\end{tabular}

Table 1: Data for etching silicon at $30 \% \mathrm{KOH}$ by weight

$\overline{\text { Where, } \mathrm{A} \text { and } \mathrm{B} \text { belongs to number of planes removed per second and time taken for removal of single }}$ plane respectively.

Tabulated data are for etch rate (ER) of silicon along three primary crystallographic orientation as they etched in $\mathrm{KOH}$ at different temperature. Results are displayed in Fig.1 which tells about ER is not a linear function with temperature. ER along three primary planes viz. (111), (110) and (100) with temperature are plotted in Fig. 1(b), by which one can easily compare their ER, which indicate (111) plane etches slow compared to other two planes: (100) and (110). 


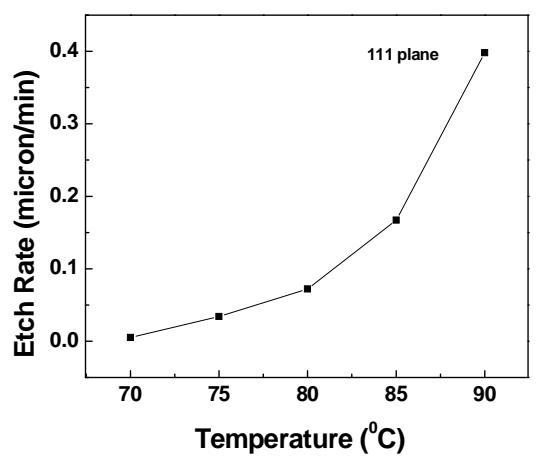

(a)

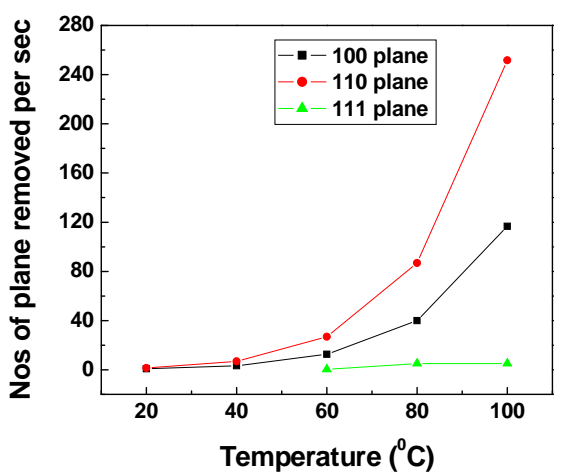

(c)

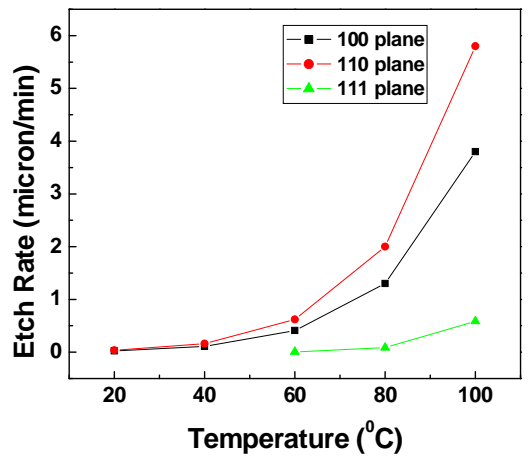

(b)

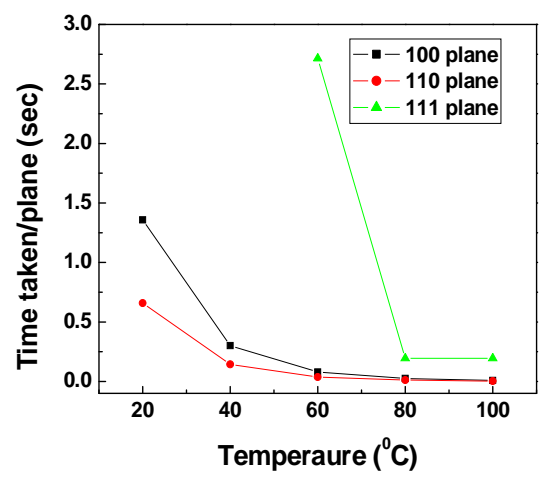

(d)

Fig 1: Various forms of anisotropic etching of silicon (a)Along (111) plane (b) Experimental (c) Nos of plane removed $\sec ^{-1}$ (d) Time for single plane removal

Simulated results reported from literature (Lee, 1969) are given in Fig. 2. It is obvious from Fig. $1 \& 2$ that ER is a function of temperature. In (111) plane ER rises little sharper beyond $80^{\circ} \mathrm{C}$ where as (110) and (100) plane is stipend before $80^{\circ} \mathrm{C}$ both in measured and simulated. Thus, it is clear that the experimental results we get are consistent with that of simulated can be taken as the sound evidence for the justification of the experiment.

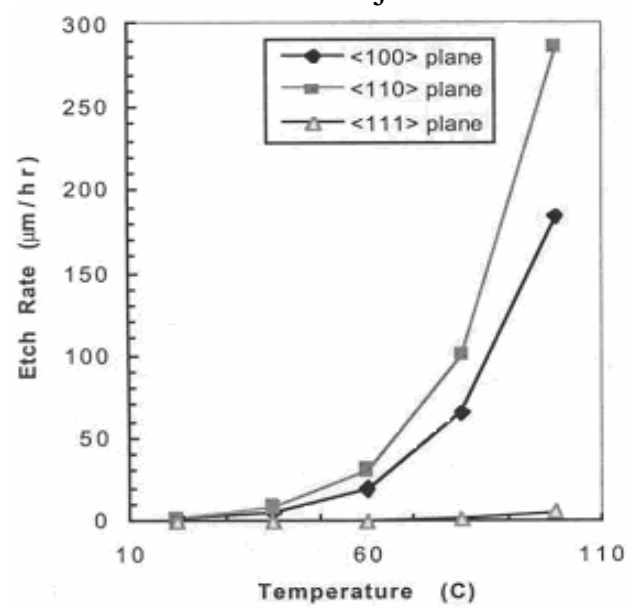

Fig 2: Simulated results of anisotropic etching of silicon 
Based on the results presented here, properties of ER in silicon are highly anisotropic. Hence, single crystal silicon has different mechanical characteristics with respect to orientation. In order to understand basic mechanism of anisotropy in silicon crystal, one should understand activation energy. Activation energy of a reaction is the amount of energy needed to start. The rate of reaction depends on the temperature at which it runs. ER and surface morphology are macroscopic results (James, 1998). As the moving surface reaches a steady state it seem to fixes etch rate. As a result, final shape of the etched wafer depends on relative etching speed along the crystallographic planes.

Etch rate (ER) depends on average of the microscopic activation energy and existence of fluctuation in fraction of particles at a fixed temperature i.e. on $p_{i}$ and $T$ (Gosalvez and Nieminen, 2003) as:

$$
E R=\frac{\Delta Z_{c . m .}}{\Delta t}=\frac{1}{\Delta t} \sum_{i}\left(\Delta Z_{c . m .}\right)_{i .} p_{i}
$$

Where $\Delta Z_{c . m}$ is distance traveled by the center of mass of the surface, $p i$ - is the removal probability of the surface atom $i$, is given by $p_{i}=p_{0 i} e^{-E_{i} / k_{B} T}$, where $T$ is temperature, $k_{B}$ is Boltzmann constant, $E_{i}$ is microscopic activation energy for removal of site $i$ and $p_{o i}$ is a prefactor. So as to understand role of temperature in microscopic energy evolution one should understand reaction mechanism takes place during etching. The chemical mechanism behind it is removal of silicon atom in $\mathrm{KOH}$ solution takes place in two steps (Wenspoek and Jansen, 1998; Ashcroft and Mermin, 1988). First, four electrons are affected in bulk silicon

$$
\mathrm{Si}+4(\mathrm{OH})^{-} \rightarrow \mathrm{Si}(\mathrm{OH})_{4}+4 e^{-}
$$

In second step, the electrons are released back into the solution accordingly

$$
4 e^{-}+4 \mathrm{H}_{2} \mathrm{O} \rightarrow 4(\mathrm{OH})^{-}+2 \mathrm{H}_{2}
$$

Products in first step $\mathrm{Si}(\mathrm{OH})_{4}$, is supposed to soluble in water. But actually, $\mathrm{Si}(\mathrm{OH})_{4}$ is decompose into water and silicon-dioxide, as a result of supplied thermal energy and hence there is removal of silicon atom with release of oxygen gas. The probability of removal of particular silicon atom depends on temperature and microscopic activation energy $\left(E_{i}\right)$ as listed above.

Above absolute zero there is gradual increase in the amplitude of atomic vibrations. As the temperature of the system increases, the number of molecules that carryout enough energy to react also increase, this means the molecules move faster and therefore collide frequently. The proportion of collision that can overcome the activation energy for the reaction increases with temperature. Phenomenon for sack out of silicon atom comes into picture if we consider microscopic activation energy as a result surface morphology is going to be modified. In this view, temperature is a factor for surface roughness. Thus, quantitative estimation of microscopic activation energy will answer these queries.

The quantitative characterization of the activation energy with temperature follows an Arrhenius nature (James, 1998) is displaced in Fig. 3. This choice is mathematically guaranteed macroscopic observation, ER, is the result of combination of microscopic activation energies of all atoms (Roher, 2001). 


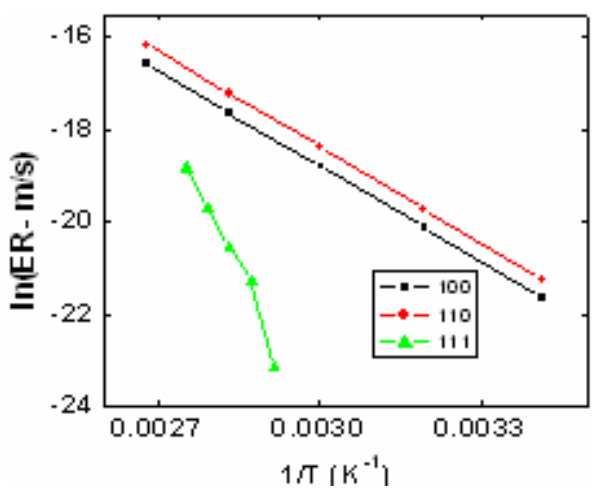

Fig 3: Anisotropic ER of silicon with its Arrhenius plot

Based on slope of Arrhenius plot, $E_{i}$, is now determined: for (111): $0.79 \mathrm{eV}$, for (110): $0.53 \mathrm{eV}$, for (100): $0.55 \mathrm{eV}$ are in the line as reported in the literature (Wang, 1999). These quantitative data justify that why ER is slow in (111) and very fast in (110), i.e., energy needed to remove a single atom is entirely different along the plane taken. Hence the plane which has higher activation energy has slow ER and vice versa. This is because (111) is closely packed; only few atoms have enough energy to cross the barrier to reach metastable state. Note that energy required to displacing an atom from its site i.e., breakdown of the bond and move to an energy crest before descending into a metastable position is associated with activation energy.

Silicon has covalent bond. If it is in excited state, activation energy must overcome to the associated covalent energy between silicon molecules. During etching, activation energy should be speed up for removal of the molecule. Cohesive energy $\left(E_{C}\right)$ of a solid is the energy required to disassemble into constituent parts i.e., its binding energy $\left(E_{B}\right)$ (Hennry, 1990) Hence, crystal like silicon, cohesive energy is the ground state binding energy. If we go down to atomic structure, cohesive energy is basically based on LCAO (Linear Combination of Atomic Orbital) model (Ashcroft and Mermin, 1980). According to this model when a crystal is formed from independent atoms, number of orbital remains same and retains much of their atomic character. However, there are continuous changes in the energies of the orbital with atomic separation. As the atoms are brought closer together, the orbits begin to interact and hence their bond strength modifies which determines ultimately the physical properties of the crystal. Based on LCAO model bond strength (Ashcroft and Mermin, 1988) is

$$
E_{B}=2 \sqrt{V_{2, h}^{2}+V_{3, h}^{2}}-\frac{1}{2} \Delta Z \cdot V_{3, h}
$$

Where, $V_{3, h}$ is hybrid polar energy, $\Delta \mathrm{Z}$ is number of valence electrons per atom and

$$
V_{2, h}=-\frac{4.37 \eta^{2}}{m d^{2}} \text { hybrid covalent energy }
$$

Each silicon atom has four valence electrons, which can form strong s-p $\mathrm{p}^{3}$ hybrid as in diamond structure (Cottam, 1989) so that energy cost for forming hybrid is s- $\mathrm{p}^{3}$ splitting. As a result, the interaction between orbital should follow inverse square law. If we consider inter-atomic separation (d) as interplanar distance then covalent energy must be cohesive energy. For known interplanar separation the cohesive energy along the crystallographic planes are now computed by equation (5) are given in the table 2 below. 
Table 2: Data for cohesive energy

\begin{tabular}{lll}
\hline \hline $\begin{array}{l}\text { Crystal } \\
\text { plane }\end{array}$ & $\begin{array}{l}\text { Interplanar } \\
\text { distance }\left({ }^{0} \mathrm{~A}\right)\end{array}$ & $\begin{array}{l}\text { Cohesive } \\
\text { energy }(\mathrm{eV})\end{array}$ \\
\hline & & \\
$(100)$ & 5.43 & 1.13 \\
$(110)$ & 3.84 & 2.25 \\
$(111)$ & 2.35 & 6.02 \\
\hline \hline
\end{tabular}

Further, it is obvious from expression (4) that bond energy decreases as the polar energy $\left(\Delta Z . V_{3, h}\right)$ increases and covalent energy decreases. Increase of $\Delta \mathrm{Z}$, decrease the bond energy and hence the cohesive energy. Cohesive energy is a function of nearest neighbor distance (Mayer, 1998). Thus, greater the cohesive energy more will be activation energy (Van, 1970). We now arrive into the conclusion that cohesive energy measures the strength of the interatomic forces and hence the stability of the crystallographic planes. This is also the evidence that less ER in (111) than other planes.

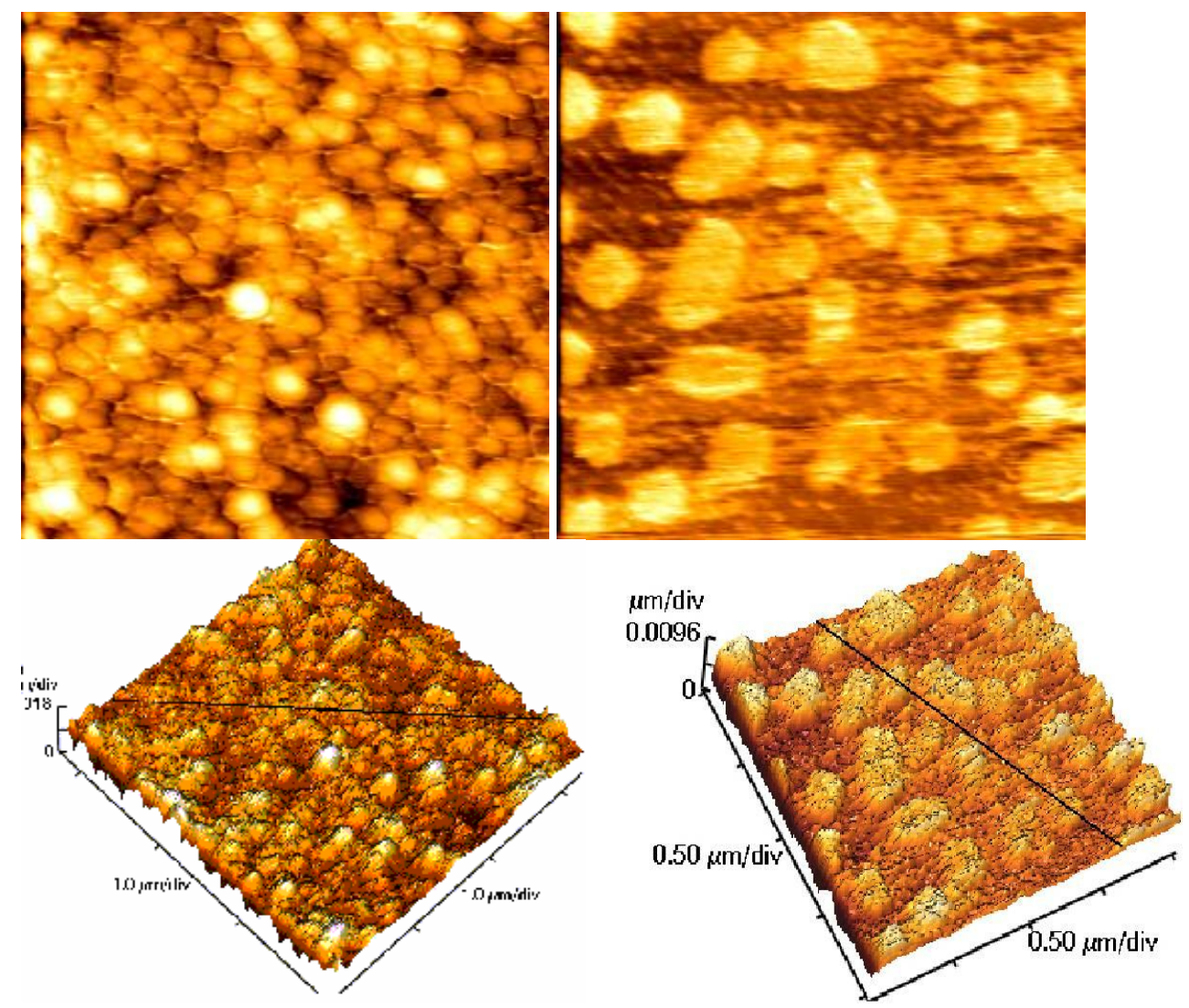

Fig 4: AFM micrographs of etched silicon at $75^{\circ} \mathrm{C}(2 \mathrm{D}$ and its $3 D)$ and at $90^{\circ} \mathrm{C}(2 \mathrm{D}$ and its $3 D)$. 


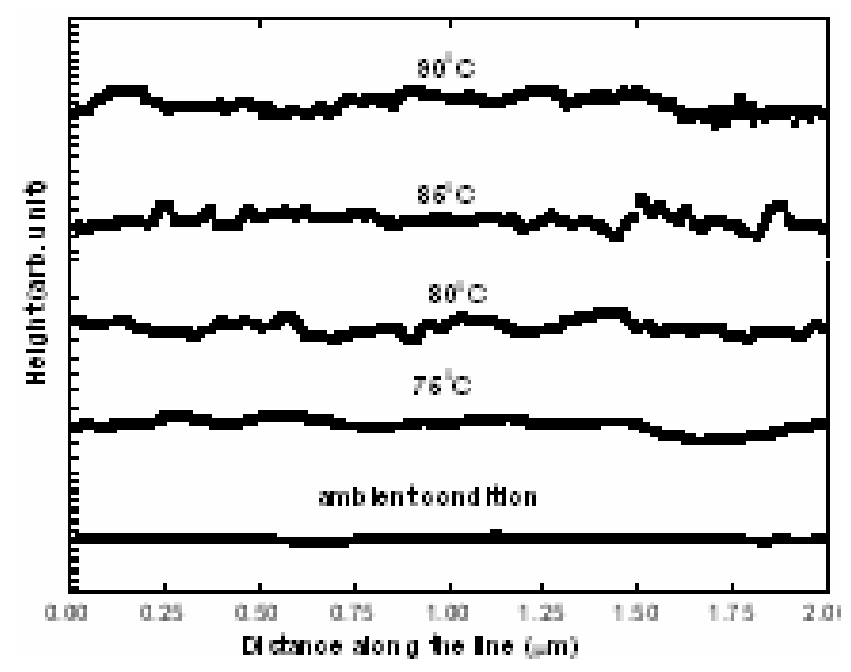

Fig. 5: Surface roughness of etched silicon at various temperatures.

AFM measurements have been employed for surface morphology at varying temperatures viz., $75^{\circ}, 80^{\circ}, 85^{\circ}$ and $90^{\circ} \mathrm{C}$. Fig. 4 summarize these results. The typical surface roughness as obtained from line analysis is also depicted. Based on the findings a smooth surface is found at room environment and rougher surface is evolved during thermal treatment. Although this etchant is relatively fast compared to other etchants, it can produce a poor surface finish as reported in literature (Abott, 1993; Schwartz and Robbin, 1961) is in the line that reported here. Thus, Fast etched planes can become covered with irregularities such as microscopic pyramids (Schwartz and Robbin, 1961).

\section{CONCLUSIONS}

Results presented here are temperature dependent ER along crystallographic orientations. This work identifies thermal agitation is a crucial factor which controls ER, activation and cohesive energies and surface morphology of the crystal plane. ER is slow in (111) face and fast in (110) face. Activation energy and cohesive energy is more in (111) and less in (100). Interplanar distance is more for (100) and less for (111) plane. Removal of silicon atom should be many particle problems and hence depends on quantum probability laws so that it follows Arrhenius behavior. Growth of surface roughness is inclined with thermal excitation. And hence, strong anisotropy in ER of $\mathrm{KOH}$ etching allow us a precious control of lateral dimension of the microstructure bound by the (111) planes to form enough foundation for miniaturized devices.

\section{ACKNOWLEDGEMENTS}

Thanks for Jamil Akhtar, Scientist CEERI, Pilani, for helping sample preparation. Thanks for Prof. P. Sen, School of Physical Sciences, JNU for the Experimentation. Thanks for University Grants Commission, Kathmandu, for fellowship to the Author.

\section{REFERENCES}

1. Kovacs, G. T. A. 1998. Micromachined Transducers Sourcebook, Boston, MA, McGraw-Hill.

2. Finne, R. M. and Klein, D. L. 1967. J. Electrochem. Soc: Solid State Science, 114: 965 
3. Campbell, S.A., Cooper, K., Dixon, L., Earwaker, R., Port, S. N. and Schiffrin, D. J. 1995. J. Micromech. Microeng. 5: 209

4. Abott A.P., Campbell S.A., Satherley, J. and Schiffrin D.J. 1993. J. Electroanal Chem. 348: 473

5. Schwartz B and Robbin H, 1961. J. Electrochem. Soc., 108365

6. Kenneth E, Bean, 1978. IEEE Transaction on Electron Devices, 25: 1185

7. Lee DB, 1969. Journal of Applied Physics, 40: 4569

8. James Marchettri, Yie He, Olaf Than, Sandeep Akkaraju, 1998. SPIES's Symposium on Micromachining and Microfabrication, Micromachimed Devices and Components, Sanate Clara, CA, USA

9. Gosalvez MA and Nieminen RM, 2003. Physical Review E 68, 031604

10. Wenspoek M and Jansen H, 1998. Silicon Micromachining, Cambridge University Press 26.

11. Ashcroft NW and Mermin ND, 1988. Sold State Physics, CBS Publishing Asia Ltd, 396

12. Roher Gregory S., 2001. Structure and Bonding in Crystalline Materials, Cambridge University Press 410

13. Wang CZ, Pan B C, and Ho K M, 1999. J. Phys. Condens. Matter 11: 2043

14. Hennry Wise and Jacques Oudar 1990. Material Concepts in Surface Reactivity and Catalysis, Academic Press Inc.

15. Cottam GM, 1989. Introduction to Surface and Super lattice Excitations, Cambridge University Press, Cambridge NY 12.

16. Mayer HP, 1998. Introduction to Solid State Physics, Viva Books Pvt. Ltd, New Delhi 426.

17. Van Vlack, 1970. Material Science for Engineers, Addison-Wesley Publishing Company, Reading, Massachusetts, 68.

18. Oosterbroek RE, Berenschot JW, Jansen V H, Nijdam AR, Pandraud G, Berg AVD and Elwenspoek MC, 2000. J. MEMS, 9: 390. 